\title{
Energy Comparison of Seven MPPT Techniques for PV Systems
}

\author{
A. DOLARA, R. FARANDA, S. LEVA \\ Department of Energy of Politecnico di Milano, Via la Masa 34, 20156, Milano, Italy. \\ Email: alberto.dolara@mail.polimi.it, \{roberto.faranda,sonia.leva\}@polimi.it \\ Received May $14^{\text {th }}, 2009$; revised July $3^{\text {rd }}, 2009$; accepted July $12^{\text {th }}, 2009$.
}

\begin{abstract}
In the future, solar energy will be a very important energy source. Several studies suppose that more than $45 \%$ of the energy in the world will be generated by photovoltaic array. Therefore it is necessary to concentrate our forces to reduce the application costs and to increment their performance. In order to reach the last aspect, it is important to note that the output characteristic of a photovoltaic array is nonlinear and changes with solar irradiation and cell's temperature. Therefore a Maximum Power Point Tracking (MPPT) technique is needed to maximize the produced energy. This paper presents a comparative study of seven widely-adopted MPPT algorithms; their performance is evaluated using, for all the techniques, a common device with minimum hardware variations. In particular, this study compares the behaviors of each technique in presence of solar irradiation variations.
\end{abstract}

Keywords: Maximum Power Point Tracking (MPPT), Photovoltaic (PV), PV Performance Comparison, Renewable Energy, DC-DC Converter.

\section{Introduction}

Solar energy is one of the most important renewable energy sources. As opposed to the conventional not renewable sources such as gasoline, coal, etc. solar energy is clean, inexhaustible and free. The main applications of photovoltaic (PV) systems are in either stand-alone (water pumping, domestic and street lighting, electric vehicles, military and space applications) [1] or grid-connected configurations (hybrid systems, power plants) [2].

Unfortunately, PV generation systems have two major problems: the conversion efficiency in electric power generation is low (in general less than 17\%, especially under low irradiation conditions), and the amount of electric power generated by solar arrays changes continuously with weather conditions.

Moreover, the solar cell V-I characteristic is nonlinear and changes with irradiation and temperature. In general, there is a point on the V-I or V-P curve only, called the Maximum Power Point (MPP), at which the entire PV system (array, inverter, etc.) operates with maximum efficiency and produces its maximum output power. The location of the MPP is not known, but can be located, either through calculation models or by search algorithms. Maximum Power Point Tracking (MPPT) techniques are used to maintain the PV array's operating point at its MPP.

Many MPPT techniques have been proposed in the lit- erature; examples are the Perturb and Observe (P\&O) method [2-5], the Incremental Conductance (IC) method [2-6], the Artificial Neural Network method [7], the Fuzzy Logic method [8], etc.. The P\&O and IC techniques, as well as variants thereof, are the most widely used.

Because of the large number of methods for MPPT, in the last years researchers and practitioners in PV systems have presented survey or comparative analysis of MPPT techniques. As a matter of fact, some papers present comparative study among only few methods $[5,6]$ and one paper presents a survey and a discussion of several MPPT methods [10]. Another paper [11] presents a ranking of ten widely adopted MPPT algorithms (P\&O, modified P\&O, Three Point Weight Comparison [12], Constant Voltage, IC, IC and CV combined [13], Short Current Pulse [14], Open Circuit Voltage [15], the Temperature Method and methods derived from it [16]), based on simulations, under the energy production point of view. The MPPT techniques are evaluated considering different types of insolation and solar irradiance variations and calculating the energy supplied by a complete PV array.

In this paper, the attention will be focused on experimental comparisons between some of these techniques, considering several irradiation conditions. Therefore, the 


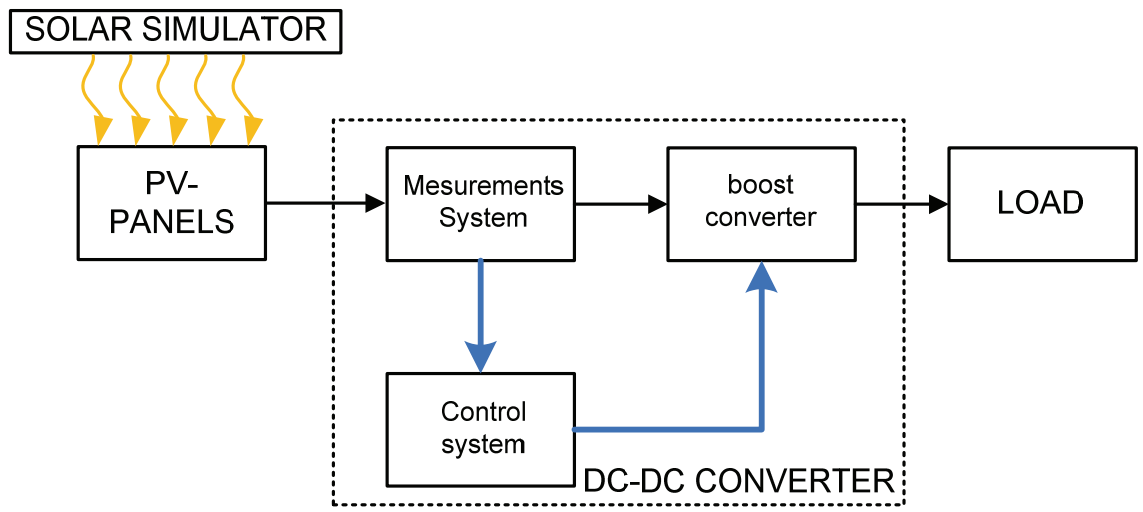

Figure 1. Block diagram of the whole experimental system

aim of this work is to compare several widely adopted MPPT algorithms between them in order to understand which technique has the best performance. The evaluation of the algorithms' performance is based on the power measurement valuating the total energy produced by the panel during the same test cycle. In this work, respect to the MPPT algorithm compared by simulations, the methods that need temperature or irradiance measurements are not considered for sake of simplicity. Indeed, as described in [11], these techniques do not have very high performance and they are too expensive. In the simulations, the considered MPPT techniques have been implemented strictly following the description indicated in the references: no MPPT algorithm is preferred and no MPPT techniques have been realized with more attention respect to the others.

In particular, without lack of generality, we will focus our attention on a stand-alone photovoltaic system constructed by connecting the DC-DC converter between the solar panel and a dc load.

\section{Experimental System}

The experimental comparison among the different MPPT techniques has been performed realizing the whole system in the Power Quality Laboratory of Department of Energy at the Politecnico di Milano.

The experimental system is constituted by three main elements (Figure 1): the DC-DC converter, the PV-panels and the solar simulator.

\subsection{The DC-DC Converter}

It has been realized a single device constituted by a DC-DC converter [17] and other components able to implement all the different MPPT techniques here analyzed, including Open Circuit Voltage (OV) [14] and Short Current Pulse (SC) [13] which required to insert further static switches to open the circuit or to create the short-circuit condition, in order to compare the results. All the MPPT techniques here described are easily ob- tained changing the software compiled in the microcontroller. In this way the differences in the measured energy load depend mainly on the software used for the implementation of the particular MPPT technique.

The choice of a stand-alone system, and hence the choice of using a DC-DC converter, reflects some industrial configurations composed by a first DC-DC conversion stage, in which usually the control of MPPT techniques is implemented, a second filter stage, and eventually a DC-AC conversion stage.

The DC-DC converter developed includes the power and control boards as shown in Figure 2.

The control board is constituted by all the components that need for the implementation of the various MPPT algorithms already illustrated in [10-16]. The microcontroller, in this case a Microchip dsPIC30f4012, is the core of the control board.

The command connection to the power board is provided by means of driver circuits which allow the valves commutation.

The interface between control and power circuits is realized with optoinsulators and Hall effect transducers to

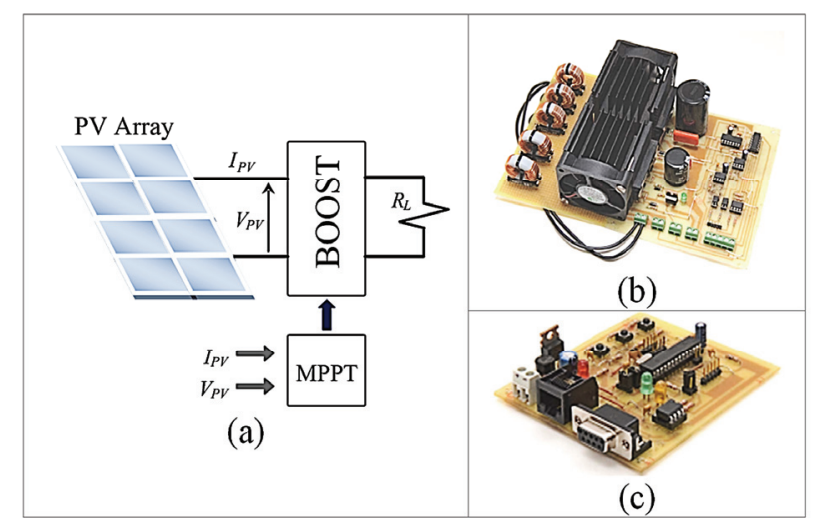

Figure 2. (a) Stand-alone PV system analyzed. DC-DC converter's (b) power and (c) control boards 
guarantee the necessary metallic insulation required between these boards. Such connection allows not only to drive the valve in PWM mode and hence to implement the different MPPT techniques without modifying the power components, but also to acquire the PV voltage and current signals.

In particular, the voltage and current measurements are made by Hall effect transducers; they are perfectly suitable for this application indeed they are able to detect continuous components, furthermore they can guarantee very low losses during the measurement and insulation between the control board and the power one, and finally they have a wide enough bandwidth.

There are a lot of DC-DC conversion circuits. In the present work the boost configuration is chosen. It is very spread thanks to its high reliability respect to other more complex configurations, to the reduced number of components and also to the high-minded experience in its operation. The complete power device scheme is shown in Figure 3.

The boost section is realized by two accumulation units, $L$ and $C_{\text {out }}$, the static switch $T_{1}$ and the diode $D_{3}$.

Moreover, the diode $D_{1}$ is put into the circuit to protect the PV-panel against negative current which could damage it.

The equivalent measures of the PV-panel voltage, $V_{P V}$, and current, $I_{P V}$, are obtained by inserting the voltage transducer $\mathrm{V}$ and the current one $\mathrm{A}$ in the circuit as reported in Figure 3.

Figure 3 shows the circuit elements $T_{v 0}, T_{s c}, K_{1}, K_{2}$, $C_{i n}$ and $D_{2}$; that have been inserted to:

- measure the PV-panel open circuit voltage, that is necessary in OV technique, through the opening of $T_{v 0}$ valve, in this case $D_{2}$ is short-circuited through $K_{2}$;

- measure the PV-panel short-circuit current, that is necessary in the $\mathrm{SC}$ technique, through the closure of $T_{s c}$ valve, in this case $T_{v 0}$ is short-circuited through $K_{1}$.

During the tests of other MPPT techniques, the valve $T_{s c}$ is kept open, while $T_{v 0}$ and $D_{2}$ are short-circuited, respectively through $K_{1}$ and $K_{2}$ switches, to increase converter efficiency removing their power losses. It is impor-

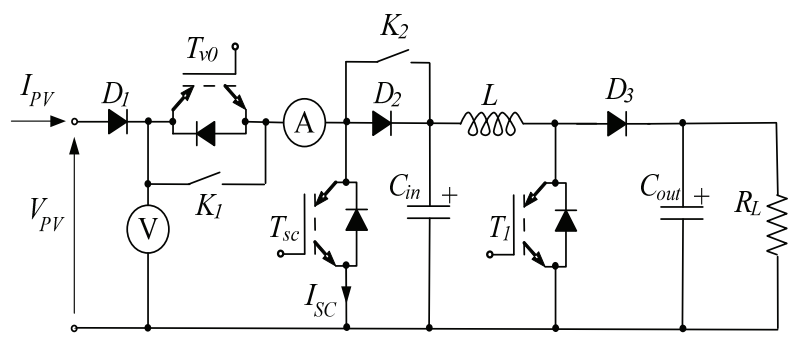

Figure 3. Scheme of the power device tant to underline that, in each MPPT algorithms, the DC-DC converter power losses do not influence the MPP because the system acquires the PV voltage and current.

It is important to note that in the SC MPPT technique it is necessary to insert the $D_{2}$ diode to avoid, during the short-circuit test, the discharging of $C_{i n}$ placed at boost input. Such capacitor is always inserted in each techniques analysed to limit the high frequency harmonic components.

The prototype converter has been sized for the voltage of 3 in-series modules and the current of 3 in-parallel modules. In particular, in correspondence of the Standard Test Condition (STC), therefore at $1000 \mathrm{~W} / \mathrm{m}^{2}$ and $298 \mathrm{~K}$, we have:

- a maximum open circuit voltage equal to $21.8 \mathrm{~V}$ and a maximum short-circuit current equal to 13.05 A with the modules in parallel configuration;

- a maximum open circuit voltage equal to $65.4 \mathrm{~V}$ and a maximum short-circuit current equal to $4.35 \mathrm{~A}$ with the modules in series configuration.

The DC-DC converter is designed to work at the MPP with a duty cycle of $25 \%$. The DC-DC converter sizing, with a security margin, leads to the following data: switching frequency of $20 \mathrm{kHz}$, nominal current of $15 \mathrm{~A}$, and nominal voltage of $150 \mathrm{~V}$.

The IGBT IRG4PC30KD electronic valves are chosen. These components integrates an ultrafast recycling diode and present small switching losses also in presence of high switching frequency.

\subsection{PV Panel}

The PV panels here considered are the poly-crystalline 70 W PV-module by Helios Technology. Its main specifications are shown in Table 1.

\subsection{Solar Simulator}

The sunlight simulator have to guarantee low spatial non-uniformity and low temporal instability of irradiance, moreover it have to generate a significant power output from PV-system and finally it have to allow different irradiance levels on the PV-panel.

The solar simulator used in the present tests is realized by using both incandescent and halogen lamps. The maximum power of the solar simulator is $2.8 \mathrm{~kW}$ and its size is $1200 \mathrm{~mm}$ long and $600 \mathrm{~mm}$ wide.

Combining the lamps, it is possible to have, with adequate uniformity, four different irradiation levels equal to $0 \mathrm{~W} / \mathrm{m}^{2}, 272 \mathrm{~W} / \mathrm{m}^{2}, 441 \mathrm{~W} / \mathrm{m}^{2}$ and $587 \mathrm{~W} / \mathrm{m}^{2}$.

\section{MPPT Control Algorithm}

There are many MPPT methods available in the literature; the most widely-used techniques are described in 
Table 1. Electrical characteristics of PV panel in STC

\begin{tabular}{ccc}
\hline Symbol & Quantity & Value \\
\hline$P_{M P P}$ & Maximum Power & $70 \mathrm{~W}$ \\
$V_{M P P}$ & Voltage at $P_{M P P}$ & $17 \mathrm{~V}$ \\
$I_{M P P}$ & Voltage at $I_{M P P}$ & $4.11 \mathrm{~A}$ \\
$I_{S C}$ & Short-Circuit Current & $4.35 \mathrm{~A}$ \\
$V_{O V}$ & Open-Circuit Voltage & $21.8 \mathrm{~V}$ \\
$N O C T$ & Nominal Operating Cell Temperature & $43 \pm 2{ }^{\circ} \mathrm{C}$ \\
\hline
\end{tabular}

the following sections, starting from the simplest method.

\subsection{Constant Voltage Method}

The Constant Voltage (CV) algorithm is the simplest MPPT control method. The operating point of the PV array is, each $n^{\text {th }}$ step, kept near the MPP by regulating the array voltage and matching it to a fixed reference voltage $V_{R E F}$ equal to the $V_{M P P}$ of the characteristic PV module (see Table 1) or another pre-valuated best voltage value [16]. The CV method assumes that insulation and temperature variations on the array are insignificant on the $V_{M P P}$, and that the constant reference voltage $V_{R E F}$ is an adequate approximation of the real MPP. Therefore, the operating point is never exactly at the MPP and different data have to be adopted for different geographical regions.

The CV method needs the measurement of the PV array voltage $V_{P V}$ in order to set up the duty-cycle $\delta$ of the DC/DC boost converter as shown in Figure 4.

It is important to observe that when the PV panel is in low insulation conditions, the $\mathrm{CV}$ technique is, generally, more effective than either the P\&O method or the IC method (analyzed below), as shown in [11]. Thanks to this characteristic, the CV method is often combined together with other MPPT techniques.

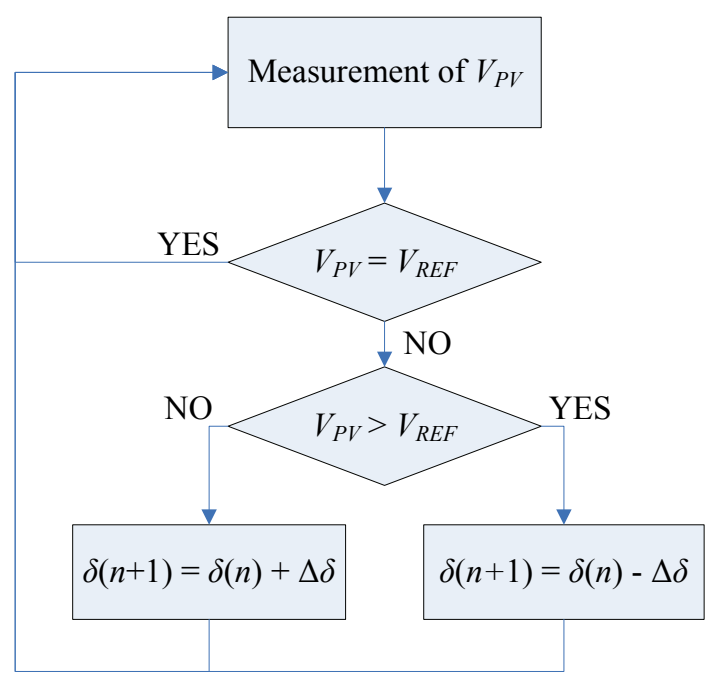

Figure 4. Flow chart of the CV method

\subsection{Short-Current Pulse Method}

The Short-Current Pulse (SC) method achieves the MPP by giving a reference current $I_{R E F}$ to the power converter controller. In fact, the optimum operating current for maximum output power is proportional to the short-circuit current $I_{S C}$ under various conditions of irradiance level $\mathrm{S}$ as follows [12]:

$$
I_{R E F}(S)=k_{1} \cdot I_{S C}(S)
$$

where $k_{l}$ is a proportional constant.

This control algorithm requires the measurement of the current $I_{S C}$. To obtain this measurement, it is necessary to introduce a static switch in parallel with the PV array, in order to create the short-circuit condition. It is important to note that when $V_{P V}=0$ no power is supplied by the PV system and consequently no energy is generated.

The SC method needs the measurement of the PV array current $I_{P V}$ in order to set up the duty-cycle of the DC/DC boost converter (see Figure ).

\subsection{Open Voltage Method}

The Open Voltage (OV) method is based on the observation that the voltage $V_{M P P}$ is always close to a fixed percentage of the open-circuit voltage $V_{O V}$. Production spread, temperature, and solar insulation levels change the position of the MPP within a $2 \%$ tolerance band. This technique uses $76 \%$ of $V_{O V}$ as reference value $V_{R E F}$ (at which the maximum output power can be obtained); in general, this value is very close to the $V_{M P P}$.

This control algorithm requires measurements of the voltage $V_{O V}$ when the circuit is opened. Here again it is necessary to introduce a static switch into the PV system; for the OV method the switch must be used to open the circuit. When $I_{P V}=0$ no power is supplied by the PV system and consequently no energy is generated. Also in this method measurement of the PV array voltage $V_{P V}$ is required by the regulator (see Figure 6).

\subsection{Perturb and Observe Methods}

The P\&O algorithms operate by periodically perturbing (i.e. incrementing or decrementing) the array terminal voltage and comparing the PV output power with that of the previous perturbation cycle. If the PV array operating 


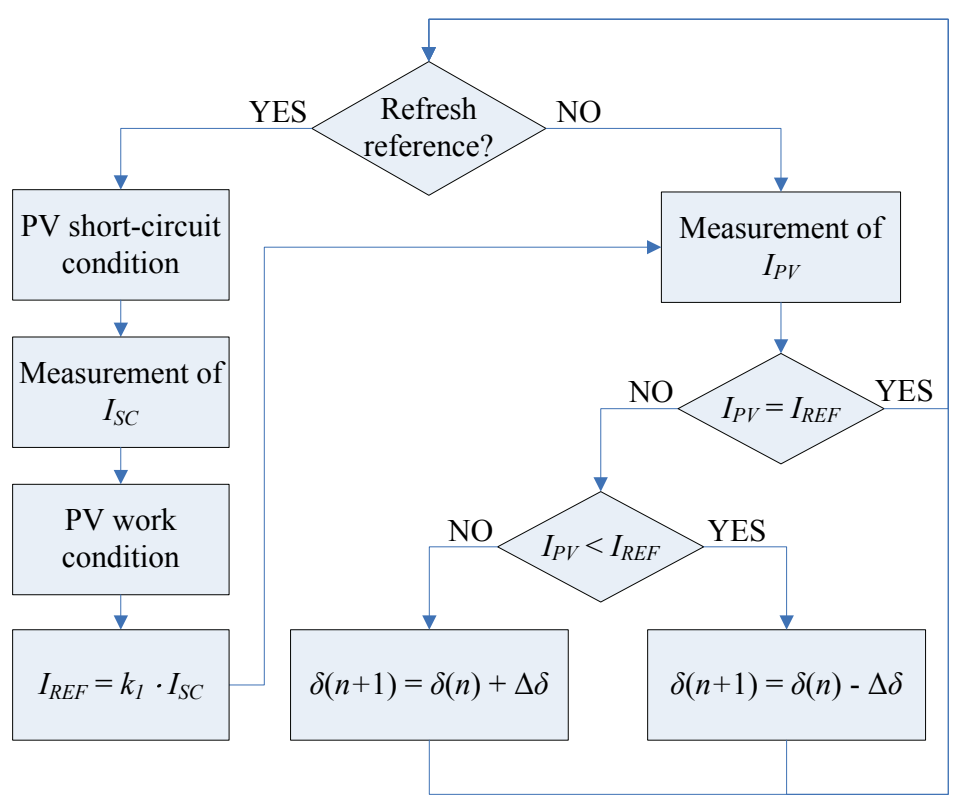

Figure 5. Flow chart of the SC method

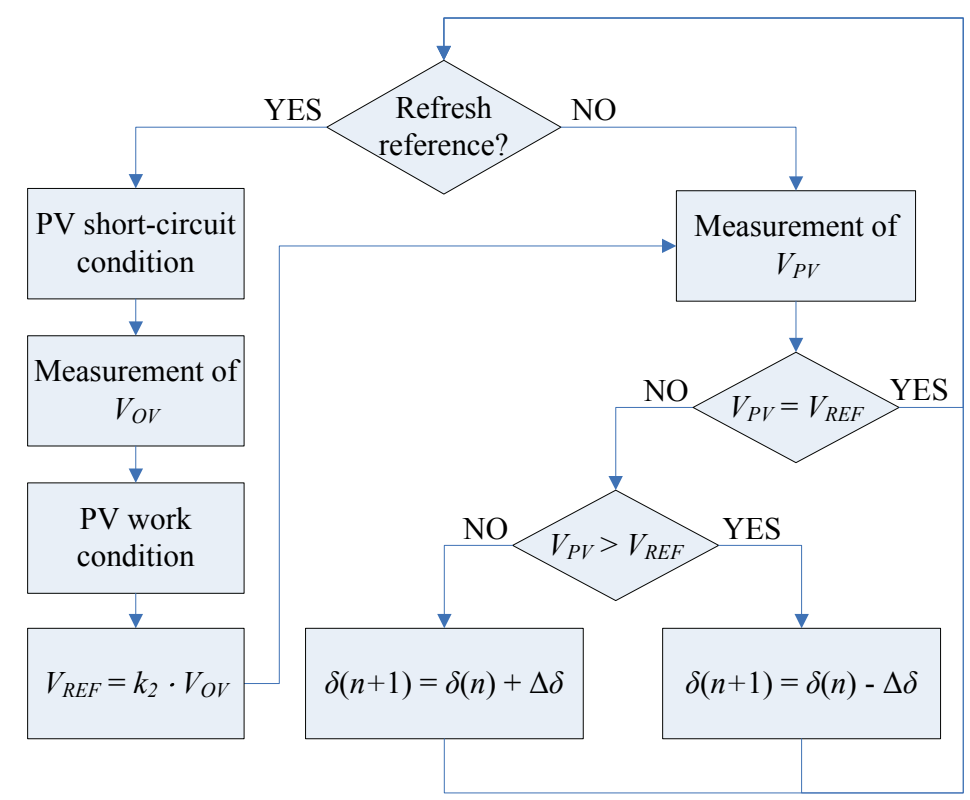

Figure 6. Flow chart of the OV method

voltage changes and power increases $\left(d P / d V_{P V}>0\right)$, the control system moves the PV array operating point in that direction; otherwise the operating point is moved in the opposite direction. In the next perturbation cycle the algorithm continues in the same way.

A common problem in $\mathrm{P} \& \mathrm{O}$ algorithms is that the array terminal voltage is perturbed every MPPT cycle; therefore when the MPP is reached, the output power oscillates around the maximum, reducing the generable power by the PV system. This is mainly true in constant or slowly-varying atmospheric conditions but also under rapidly changing atmospheric conditions [17].

There are many different $\mathrm{P} \& \mathrm{O}$ methods available in the literature. In this paper we consider the classic, the optimized and the three-points $\mathrm{P} \& \mathrm{O}$ algorithms.

In the classic $\mathrm{P} \& \mathrm{O}$ technique $(\mathrm{P} \& \mathrm{Oa})$, the perturba- 


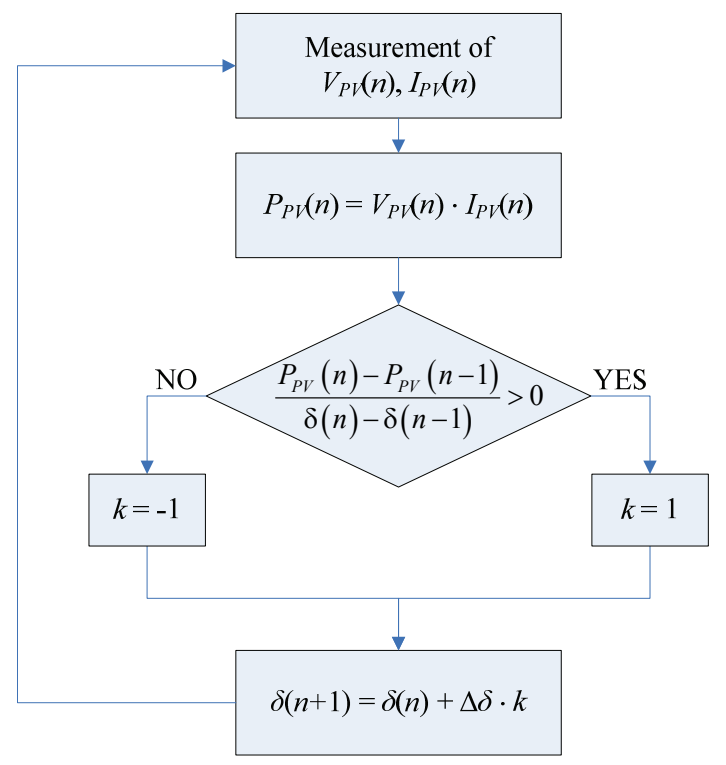

Figure 7. Flow chart of the P\&Oa method

tions of the PV operating point have a fixed magnitude (see Figure 7). In our analysis, the magnitude of perturbation $\Delta \delta$ is $0.35 \%$ of $\mathrm{PV}$ array $V_{O V}$. In the optimized $\mathrm{P} \& \mathrm{O}$ technique $(\mathrm{P} \& \mathrm{Ob})$, an average of several samples of the array power is used to dynamically adjust the magnitude of the perturbation of the PV operating point. This adjusting is realized by valuating $M_{O V}$ and calculating the parameter $a(n)$ as reported in Figure 8. In the three-point weight comparison method (P\&Oc), the perturbation direction is decided by comparing the PV output power on three points of the $P-V$ curve, and valuating the parameter $M$ as shown in Figure 9. These three points are the current operation point $\mathrm{A}$, a point $\mathrm{B}$ perturbed from point $\mathrm{A}$, and a point $\mathrm{C}$ doubly perturbed in the opposite direction from point $\mathrm{B}$.

All these three algorithms require the measurement of the PV array voltage $V_{P V}$ and of the PV array current $I_{P V}$.

\subsection{Incremental Conductance Method}

The Incremental Conductance (IC) algorithm is based on the observation that the following equation holds at the MPP [2]:

$$
\left(d I_{P V} / d V_{P V}\right)+\left(I_{P V} / V_{P V}\right)=0
$$

where $I_{P V}$ and $V_{P V}$ are the PV array current and voltage, respectively. When the operating point in the $P-V$ plane is to the right of the MPP, it is verified $\left(d I_{P V} / d V_{P V}\right)+$ $\left(I_{P V} / V_{P V}\right)<0$, whereas when it is to the left of the MPP this $\left(d I_{P V} / d V_{P V}\right)+\left(I_{P V} / V_{P V}\right)>0$.

The MPP can thus be tracked by comparing the instantaneous conductance $I_{P V} / V_{P V}$ to the incremental conductance $d I_{P V} / d V_{P V}$. Therefore, if the quantity $\left(d I_{P V} / d V_{P V}\right)$ $+\left(I_{P V} / V_{P V}\right)$ is more than $\varepsilon$, its sign means a power production decrement and indicates the correct direction of per- turbation leading to the MPP. Once MPP has been reached, the operation of $\mathrm{PV}$ array is maintained at this point and the perturbation stopped unless a change in $d I_{P V}$ is noted. In this case, the algorithm decrements or increments the PV array voltage $V_{P V}$ to track a new MPP. The increment size determines how fast the MPP is tracked.

The IC method offers good performance under rapidly changing atmospheric conditions. The classic IC algorithm requires the measurement of the PV array voltage $V_{P V}$ and current $I_{P V}$ in order to determine the correct perturbation direction.

\section{Numerical Results}

The measurements have been performed several times in order to cut off deviations caused by interferences and/or environmental factors in this system. The most important environmental factor, that hardly influence the PV-panel behaviour, is its temperature. In order to maintain the PV-panel temperature equal in all tests and to preserve this parameter into a little range during tests, all experiments are made starting from the same PV-panel's temperature, and the duration of tests has been reduced as short as possible avoiding overheating.

Due to energy absorbed from the network, available space constraints and especially economic constraints associated to the dimensions of the solar simulator, the test campaign involved a single module described in Table 1.

In order to realize a precise analysis of the performance of the different MPPT techniques, they are experimentally compared taking into account two different irradiation diagrams. The first one, Case 1 (Figure 11), is characterized by medium and medium-high irradiation levels of $441 \mathrm{~W} / \mathrm{m}^{2}$ and $587 \mathrm{~W} / \mathrm{m}^{2}$ with a time of $180 \mathrm{~s}$ and the second one, Case 2 (Figure 12), with low, lo$\mathrm{W}$-medium, medium-high irradiation levels of $0 \mathrm{~W} / \mathrm{m}^{2}$, $272 \mathrm{~W} / \mathrm{m}^{2}, 441 \mathrm{~W} / \mathrm{m}^{2}$ and $587 \mathrm{~W} / \mathrm{m}^{2}$, with a time of $160 \mathrm{~s}$ (Case 2 include a $10 \mathrm{~s}$ interval without irradiation).

Every MPPT technique analysis starts when the initial steady state condition of each case is reached.

A couple of samples of voltage and current is available every $10 \mathrm{~ms}$, and $\mathrm{P} \& \mathrm{Oa}, \mathrm{P} \& \mathrm{Ob}, \mathrm{IC}, \mathrm{CV}, \mathrm{OV}$ and SC algorithms can perform an iteration for each couple of values; only P\&Oc needs 3 measurement of power instead of 1 .

Duty-cycle variation amplitude $\Delta \delta$ is $0.5 \%$ for all techniques except $\mathrm{P} \& \mathrm{Ob}$, in witch $\Delta \delta$ is proportional to the ratio $d P / d V$ and it ranges between $0.5 \%$ and $2.7 \%$. A reduced duty-cycle variation value decreases the speed of the algorithm dynamic behaviour but it increases the precision in reaching MPP.

The P\&Oa technique performs very well with low radiance values: in this condition the P-V curve is very smooth near the maximum. The $\mathrm{P} \& \mathrm{Ob}$ logic with variable step is able to reduce steady state oscillations and, at the same time, to provide higher response speeds at me- 


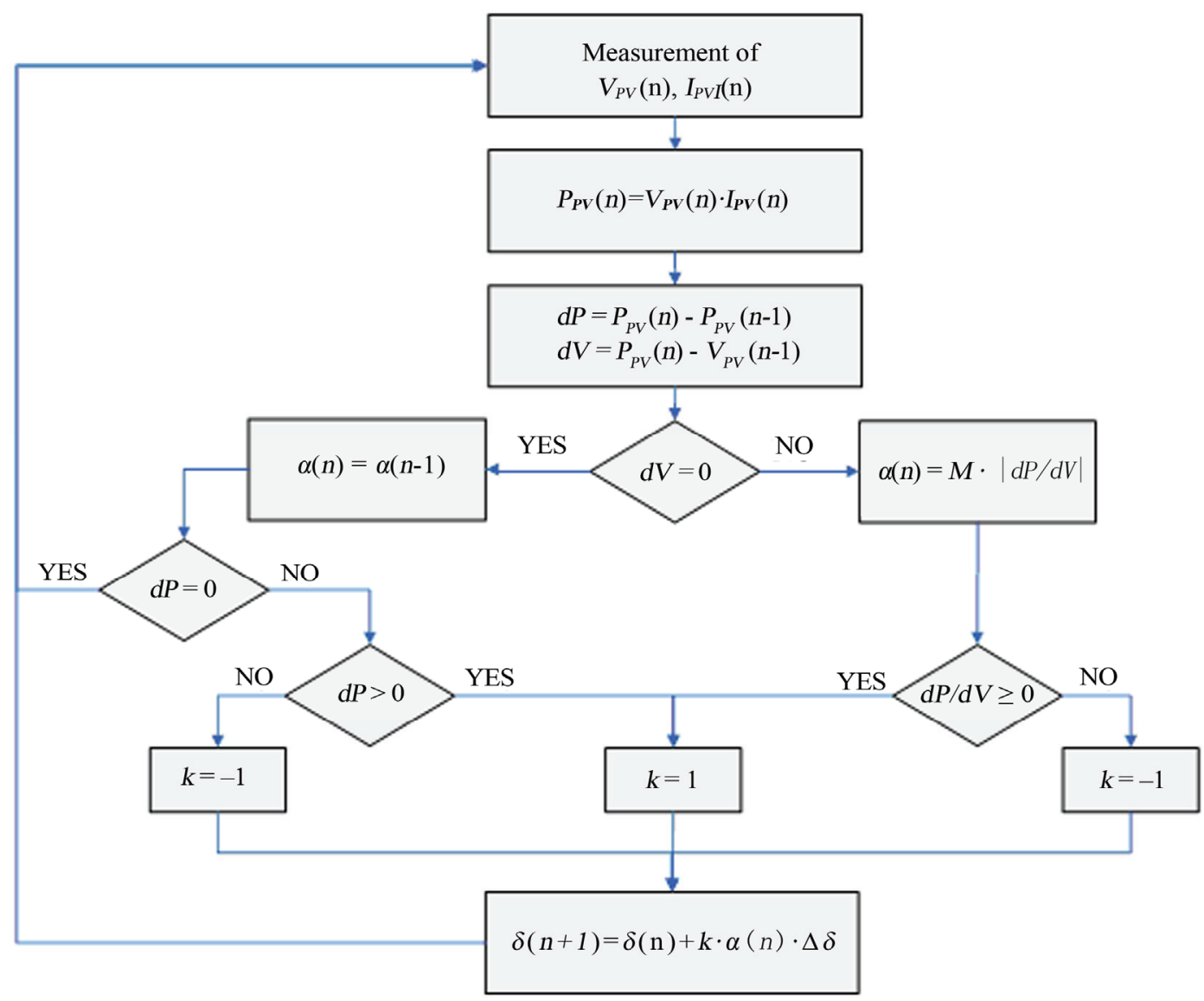

Figure 8. Flow chart of the optimized P\&Ob method

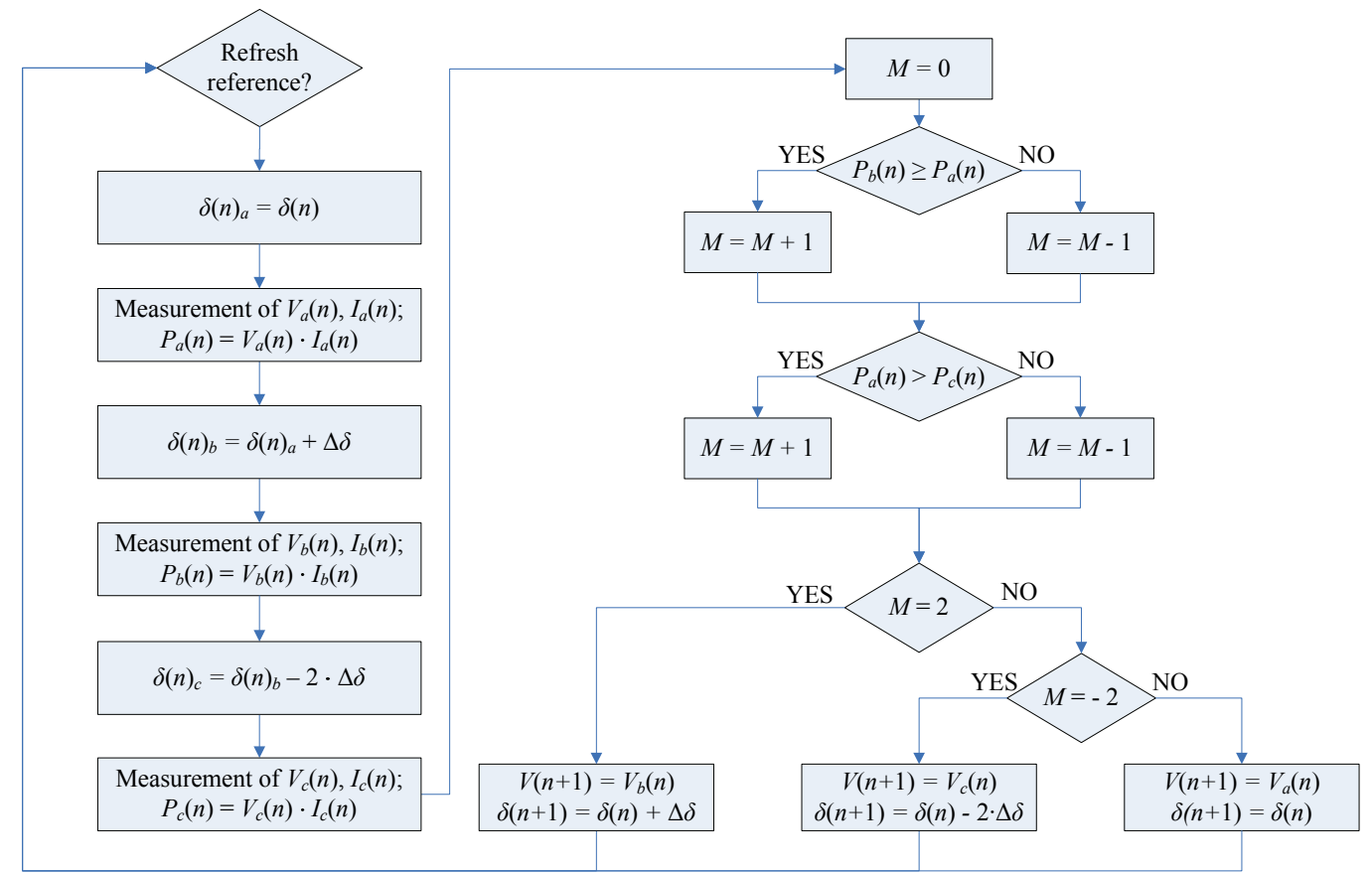

Figure 9. Flow chart of the optimized P\&Oc method 


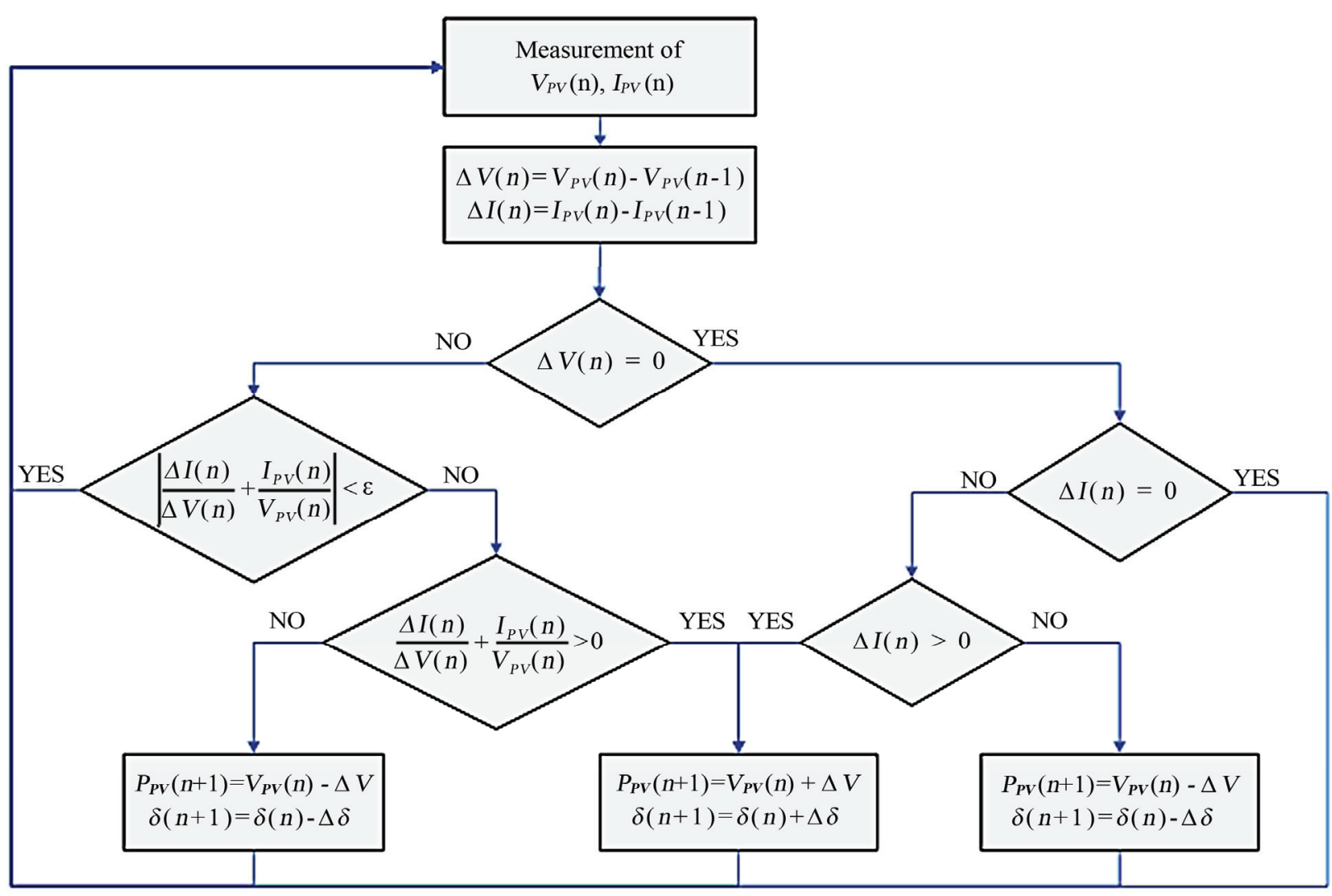

Figure 10. Flow chart of the IC method

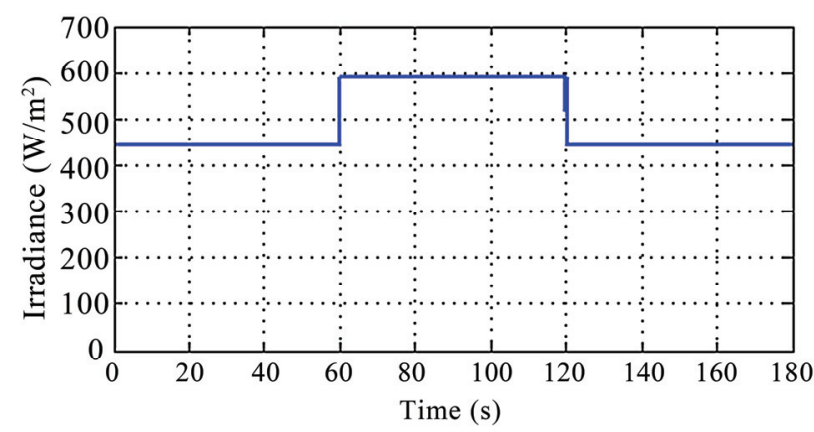

Figure 11. Irradiation diagram of Case 1

dium-high irradiance level with respect to the P\&Oa approach with fixed $\Delta \delta$. This technique is very slow in reaching MPP when irradiance level is low because $d P / d V$ is small.

The P\&Oc technique compare the power of three different working points as described in Fig. 9. The algorithm modifies the duty-cycle, in function of the obtained results, to reach the MPP value as described in [12]. The increment (or decrement) of the duty-cycle amplitude is constant and the algorithm performs an iteration every $10 \mathrm{~ms}$.

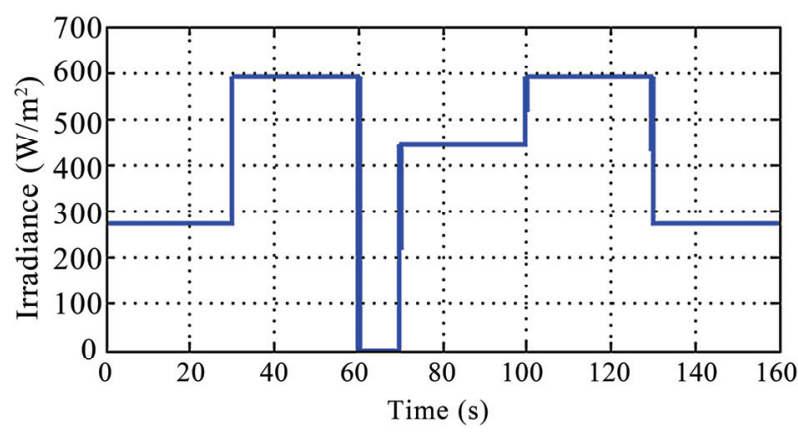

Figure 12. Irradiation diagram of Case 2

Case 2 shows the main disadvantage of the IC technique: for low radiance values the technique works on a $\mathrm{P}-\mathrm{V}$ curve with a derivative close to zero in a large interval around the maximum value, therefore it is not able to properly identify the MPP. It results in oscillations around the MPP with a reduced output energy value.

The CV technique is optimized for a single radiance value; the performance of this technique is strongly related with the voltage set point. It provides satisfying results, but they are not as good as the ones provided by $\mathrm{P} \& \mathrm{O}$ and IC techniques. 
MPPT Technique

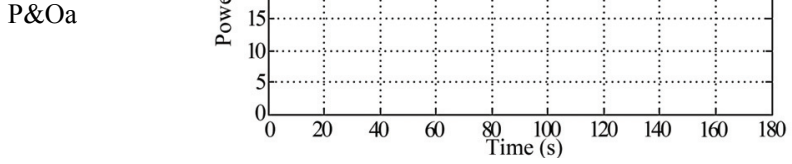

$\mathrm{P} \& \mathrm{Ob}$

IC

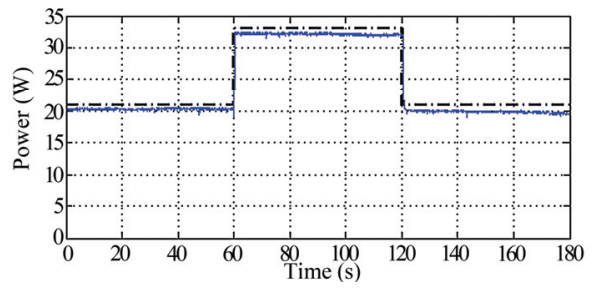

$\mathrm{P} \& \mathrm{Oc}$
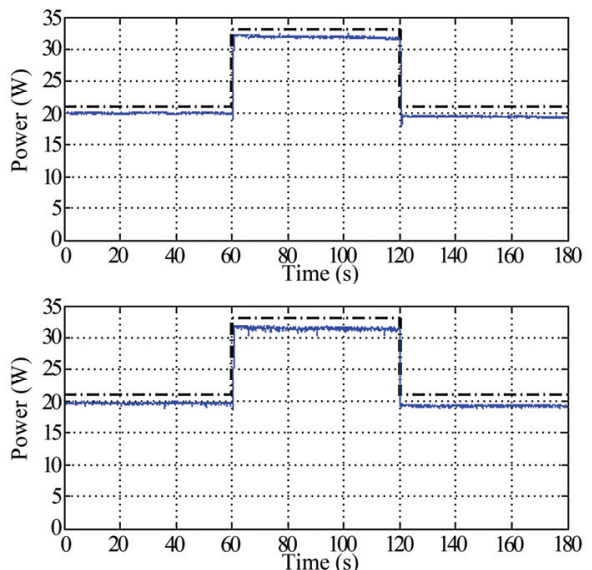

CV
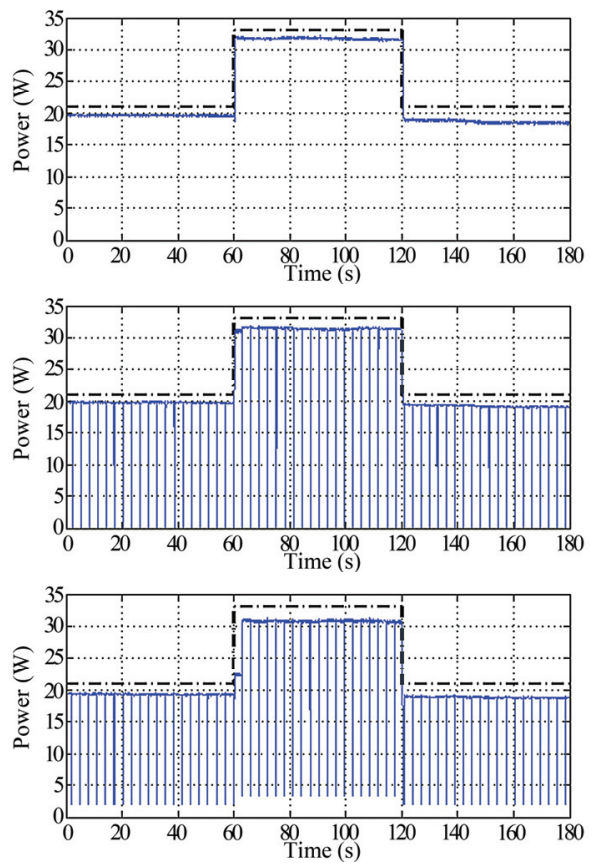

Case 2
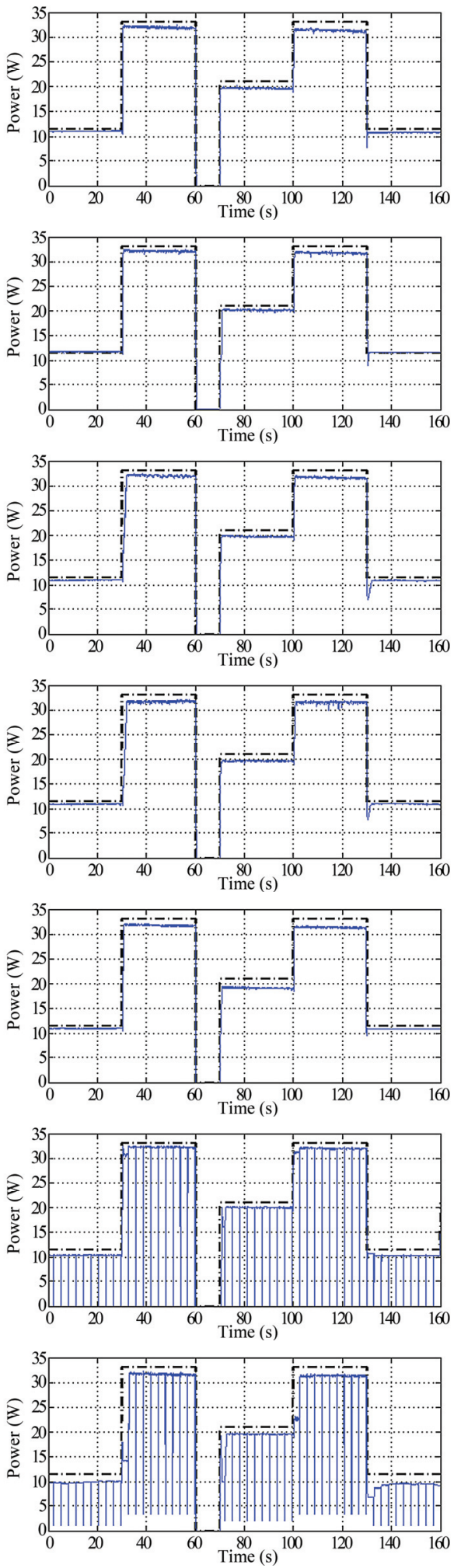

Figure 13. Power generated by the PV array in the Case 1 and Case 2 by different optimized MPPT methods (solid line) and ideal (dot-dashed line) MPPT method 
Further considerations may regard $\mathrm{OV}$ and $\mathrm{SC}$ techniques. They require additional valves for, respectively, the measurement of the PV open circuit voltage and of the PV short-circuit current.

Concerning the OV technique, it refreshes the voltage reference value every $3 \mathrm{~s}$ through the open voltage measurement (for this measurement is necessary $10 \mathrm{~ms}$ without power generation). The ratio of the open voltage and the MPP voltage is not strictly constant with temperature, and the technique can be optimized only for a single temperature value. For this reason the converter performance with $\mathrm{OV}$ technique is in general better than $\mathrm{CV}$, but is not as good as the ones provided by $\mathrm{P} \& \mathrm{O}$ and IC techniques because of the voltage drop on $T_{v 0}$ and the necessary measurement time. It is important to underline that the voltage drop on $T_{v 0}$ is equal to about $1.5 \mathrm{~V}$, which implies a significant reduction of the load voltage, and hence of the output power. This is a significant feature in the evaluation of the converter efficiency.

Analogous considerations regard the SC technique. In this case the voltage drop that reduces converter's output power is due to the $D_{2}$ valve and it is about $0.6 \mathrm{~V}$. This technique refreshes the reference current value once again every $3 \mathrm{~s}$ through the short-circuit current measurement (for this measurement is necessary $10 \mathrm{~ms}$ without power generation). The voltage applied to the $\mathrm{PV}$-panel during the measurement step is the voltage drop on $T_{s c}$. In this condition the measured current can be approximated to the real short-circuit current.

Figure 13 shows the power generated from the PV-panel with the same converter configuration and different MPPT techniques in the two cases. The diagrams also show the ideal power, obtained by using an ideal MPPT technique that is equal to the maximum power that the PV-panel can produce. These values are measured directly on the PV-panel under test in STC. For this reason MPPT ideal curve must be considered only as a qualitative reference to compare tests' results. It is important to observe that the uncertain in the PV voltage and current measurements and the small difference in the environmental conditions between each tests suggest that the results cannot be a good reference to calculate the efficiency of the single MPPT algorithm. In these conditions, even an uncertain of $0.5 \%$ in the measurements could produce an uncertain in the relative power losses that could be more than $10 \%$.

Table 2 summarizes the performances of the different techniques in the two radiation cases and the differences respect to the ideal MPPT algorithm.

\section{Conclusions}

This paper has presented a comparison among some of the more diffused Maximum Power Point Tracking techniques in relation to their energy performance. In particular, different types of solar insolation characterized by low and medium irradiation level are considered, and the energy supplied by a complete PV array is experimentally evaluated. The whole system-including the DC-DC converter and the lighting system - is arranged in the Power Quality Laboratory of Department of Energy of the Politecnico di Milano. The different MPPT techniques have been implemented following the directions indicates in the papers listed in the references; no one has been preferred or better improved respect to the others.

The results show that the best MPPT technique is the modified $\mathrm{P} \& \mathrm{O}(\mathrm{P} \& \mathrm{Ob})$. The logic turned out to be effective in both the situations here considered, providing always the highest efficiency. $\mathrm{P} \& \mathrm{Ob}$ technique shows its limit in the response to the irradiance variation at low irradiance level.

The IC technique has an efficiency lower than the $\mathrm{P} \& \mathrm{O}$ techniques, but its response time is quite independent to the irradiation values and its efficiency increase with the irradiance level. This technique can be a good alternative to the $\mathrm{P} \& \mathrm{O}$ techniques in applications characterized by high, fast and continuous radiance variations, e.g. the PV applications in transportation.

The two techniques are also equivalent concerning the costs and the software complexity; in particular both the techniques require a microcontroller with medium/higher performances than the ones required by other techniques, due to the necessity of high computation capability.

Among the other hill climbing techniques, the $\mathrm{P} \& \mathrm{Oa}$ method presents acceptable results: this algorithm can be a good alternative to the two previous techniques. Instead the P\&Oc method, even if characterized by output energy values analogous to the $\mathrm{P} \& \mathrm{Oa}$, has a more complex algorithm and a lower reactivity, with no benefit in terms of performances. Furthermore, given the features required by the controller, the $\mathrm{P} \& \mathrm{Ob}$ technique is better than the $\mathrm{P} \& \mathrm{Oc}$ one.

The $\mathrm{P} \& \mathrm{O}$ a technique requires a microcontroller which has lower computational capability constraints with respect to the best technique here considered. It is therefore necessary to evaluate if the cost gap between the two microcontrollers can justify the lower performances of the technique.

It is necessary to underline that the maximum irradiance level obtained from solar simulator is about half than the real irradiance from the sun. In these conditions the performance of IC are quite less than the $\mathrm{P} \& \mathrm{O}$ techniques ones because the MPP in the PV power characteristic has a derivative close to zero for a quite large voltage variation.

In the present analysis the $\mathrm{CV}, \mathrm{OV}$ and $\mathrm{SC}$ techniques turned out to be the worst ones. Their performances are lower than the ones obtained with P\&Ob techniques especially in case of conditions very different from the radiance value in correspondence of which these techniques have been modeled. Moreover, OV and SC techniques 
Table 2. Energy generated as a function of MPPT technique and irradiance input

\begin{tabular}{|c|c|c|c|c|c|c|}
\hline $\begin{array}{c}\text { MPPT } \\
\text { Technique }\end{array}$ & \multicolumn{3}{|c|}{ Case 1} & \multicolumn{3}{|c|}{ Case 2} \\
\hline & Energy $[\mathrm{J}]$ & Rank & $\begin{array}{l}\text { Difference respect to } \\
\text { the ideal case }\end{array}$ & Energy $[\mathrm{J}]$ & Rank & $\begin{array}{l}\text { Difference respect } \\
\text { to the ideal case }\end{array}$ \\
\hline Ideal & 4493 & - & - & 3298 & - & - \\
\hline $\mathrm{P} \& \mathrm{Oa}$ & 4282 & 2 & $-4,7 \%$ & 3144 & 2 & $-4,7 \%$ \\
\hline $\mathrm{P} \& \mathrm{Ob}$ & 4346 & 1 & $-3,3 \%$ & 3212 & 1 & $-2,6 \%$ \\
\hline $\mathrm{P} \& \mathrm{Oc}$ & 4278 & 3 & $-4,8 \%$ & 3135 & 3 & $-4,9 \%$ \\
\hline IC & 4215 & 4 & $-6,2 \%$ & 3117 & 4 & $-5,5 \%$ \\
\hline $\mathrm{CV}$ & 4201 & 5 & $-6,5 \%$ & 3100 & 6 & $-6,0 \%$ \\
\hline $\mathrm{OV}$ & 4200 & 6 & $-6,5 \%$ & 3104 & 5 & $-5,9 \%$ \\
\hline $\mathrm{SC}$ & 4088 & 7 & $-9,0 \%$ & 2942 & 7 & $-10,8 \%$ \\
\hline
\end{tabular}

requires additional valves in the converter that decrease its efficiency and the output power.

The CV technique is still a very simple logic which provides a very good efficiency for radiance values closed to $700 \mathrm{~W} / \mathrm{m}^{2}$, with low costs. Hence, generally this technique can be selected only if there is the necessity to minimize the control system cost.

However the cost of a microcontroller currently low, so that the implementation of the $\mathrm{P} \& \mathrm{O}$ type techniques is anyway preferred.

\section{REFERENCES}

[1] J. Schaefer, "Review of photovoltaic power plant performance and economics," IEEE Trans. Energy Convers., EC-5, pp. 232-238, 1990.

[2] N. Femia, D. Granozio, G. Petrone, G. Spaguuolo, and M. Vitelli, "Optimized one-cycle control in photovoltaic grid connected applications," IEEE Trans. Aerosp. Electron. Syst., Vol. 42, pp. 954-972, 2006.

[3] W. Wu, N. Pongratananukul, W. Qiu, K. Rustom, T. Kasparis, and I. Batarseh, "DSP-based multiple peack power tracking for expandable power system," in Proc. APEC, pp. 525-530, 2003.

[4] C. Hua and C. Shen, "Comparative study of peak power tracking techniques for solar storage system," in Proc. APEC, pp. 679-685, 1998.

[5] D. P. Hohm and M. E. Ropp, "Comparative study of maximum power point tracking algorithms using an experimental, programmable, maximum power point tracking test bed," in Proc. Photovoltaic Specialist Conference, pp. 1699-1702, 2000.

[6] K. H. Hussein, I. Muta, T. Hoshino, and M. Osakada, "Maximum power point tracking: an algorithm for rapidly chancing atmospheric conditions," IEE Proc.-Gener. Transm. Distrib., Vol. 142, pp. 59-64, 1995.

[7] X. Sun, W. Wu, X. Li, and Q. Zhao, "A research on photovoltaic energy controlling system with maximum power point tracking," in Power Conversion Conference, pp. 822-826, 2002.
[8] T. L. Kottas, Y. S. Boutalis, and A. D. Karlis, "New maximum power point tracker for PV arrays using fuzzy controller in close cooperation with fuzzy cognitive network," IEEE Trans. Energy Conv., Vol. 21, pp. 793-803, 2006.

[9] I. S. Kim, M. B. Kim, and M. Y. Youn, "New maximum power point tracking using sliding-mode observe for estimation of solar array current in the grid-connected photovoltaic system,” IEEE Trans. Ind. Electron., Vol. 53, pp. 1027-1035, 2006.

[10] Y. T. Hsiao and C. H. Chen, "Maximum power tracking for photovoltaic power system," in Proc. Industry Application Conference, pp. 1035-1040, 2002.

[11] G. J. Yu, Y. S. Jung, J. Y. Choi, I. Choy, J. H. Song, and G. S. Kim, "A novel two-mode MPPT control algorithm based on comparative study of existing algorithms," in Proc. Photovoltaic Specialists Conference, pp. 1531-1534, 2002.

[12] T. Noguchi, S. Togashi, and R. Nakamoto, "Short-current pulse-based maximum-power-point tracking method for multiple photovoltaic-and-converter module system," IEEE Trans. Ind. Electron., Vol. 49, pp. 217-223, 2002.

[13] D. Y. Lee, H. J. Noh, D. S. Hyun, and I. Choy, "An improved MPPT converter using current compensation method for small scaled PV-applications," in Proc. APEC, pp. 540-545, 2003.

[14] M. Park and I. K. Yu, "A study on optimal voltage for MPPT obtained by surface temperature of solar cell," in Proc. IECON, pp. 2040-2045, 2004.

[15] T. Takashima, T. Tanaka, M. Amano, and Y. Ando, "Maximum output control of photovoltaic (PV) array," in Proc. 35th Intersociety Energy Convers. Eng. Conf. Exhib., pp. 380-383, 2000.

[16] P. C. M. de Carvalho, R. S. T. Pontes, D. S. Oliveira, D. B. Riffel, R. G. V. de Oliveira, and S. B. Mesquita, "Control method of a photovoltaic powered reverse osmosis plant without batteries based on maximum power point tracking," in Proc. IEEE/PES Transmiss. Distrib. Conf. Expo.: Latin America, pp. 137-142, 2004.

[17] T. Esram and P. L. Chapman, "Comparison of photovoltaic array maximum power point tracking techniques," IEEE Trans. Energy Conv., Vol. 22, pp. 439-449, 2007. 